ISSN: 0213-2052 - eISSN: 2530-4100

DOI: https://doi.org/10.14201/shha202038139150

\title{
UN ALTAR INÉDITO PROCEDENTE \\ DE HERGUIJUELA DE LA SIERRA (SALAMANCA) Y NUEVA INTERPRETACIÓN DE TRES ARAS VOTIVAS EXTREMEÑAS
}

\section{One Unpublished Altar from Herguijuela de la Sierra (Salamanca) and a New Interpretation on Three Votive Altars from Extremadura}

\author{
Juan Carlos OLIVARES PEDREÑO \\ Universidad de Alicante \\ jc.olivares@ua.es
}

Fecha de recepción: 2-7-2019; aceptación definitiva: 4-05-2020

https://orcid.org/0000-0002-8146-4901

RESUMEN: En el presente trabajo presentamos un altar votivo inédito hallado al sur de la provincia de Salamanca, en la región de la Sierra de Francia, y también proponemos cambios de lectura en dos inscripciones votivas procedentes de las localidades extremeñas de Salvatierra de los Barros (Badajoz) y Santa Ana (Cáceres), además de la revisión de otro altar de procedencia desconocida, que está conservado en la actualidad en el Museo de Badajoz. En la primera de estas inscripciones ya publicadas proponemos cambios en el teónimo citado en la pieza; en la segunda desciframos los nombres de los oferentes y en la última proponemos cambios menos importantes, pero necesarios para obtener la lectura precisa del monumento.

Palabras clave: Epigrafía; aras votivas; religión; Lusitania. 
ABSTRACT: In the present work we study an unpublished votive altar found in the south of the province of Salamanca, in the region of the Sierra de Francia, and we also propose changes of reading in two votive inscriptions coming from the Extremadura villages of Salvatierra de los Barros (Badajoz) and Santa Ana (Cáceres). Besides, we analize another altar of unknown origin, which is currently preserved in the Badajoz Museum. In the first of these inscriptions already published, we propose changes in the theonym quoted in the piece; in the second, we decipher the names of the dedicants and in the last one, we propose less important changes, but necessary to obtain the accurate reading of the monument.

Keywords: Epigraphy; votive altars; religion; Lusitania.

El presente estudio es resultado de varios viajes que llevamos a cabo a tierras extremeñas y al sur de la provincia de Salamanca durante el último año, con el objetivo de revisar, sobre el terreno, las lecturas de diversas inscripciones votivas dedicadas a divinidades paleohispánicas. Esta labor la desarrollamos para intentar dar mayor fiabilidad a la base documental disponible sobre los cultos indígenas hispanos de los territorios lusitano y vetón, lo que permitirá en el futuro establecer interpretaciones más sólidas y mejor fundamentadas.

\section{Ara votiva inédita hallada en Hergujuelda de la Sierra, Salamanca}

El ara votiva que presentamos como inédita se encontró durante la reforma del edificio del Ayuntamiento de Herguijuela de la Sierra, en la Sierra de Francia. La pieza es de granito. Conserva un bocel de la moldura superior y ha perdido la cornisa y su zócalo por completo, además de parte de la zona inferior del fuste que, al parecer, no ha afectado al texto. Al no ser visible el posible focus ni la forma de la cabecera y el zócalo con seguridad, formalmente no se puede asegurar plenamente que se trate de un altar, pero nos llevan a esa conclusión el texto y la existencia de la citada moldura superior. También acusa un cierto desgaste el campo epigráfico en la línea 2 del texto, lo que dificulta la lectura. No sabemos si el ara está trabajada a cuatro caras por estar insertada en una pared. Las medidas totales de la pieza son (43) x (28) $\mathrm{cm}$. Las letras son capitales rústicas y actuarias, con medidas entre 4,6 y $6 \mathrm{~cm}$. No se observan interpunciones.

El monumento, hallado durante las obras de restauración del edificio del Ayuntamiento de la localidad, se insertó en la pared frontal del mismo, en el porche que da a la plaza de España, a dos metros de altura, donde 
Y NUEVA INTERPRETACIÓN DE TRES ARAS VOTIVAS EXTREMEÑAS

se encuentra en la actualidad y donde la pudimos estudiar y fotografiar el 19 de junio de $2018^{1}$.

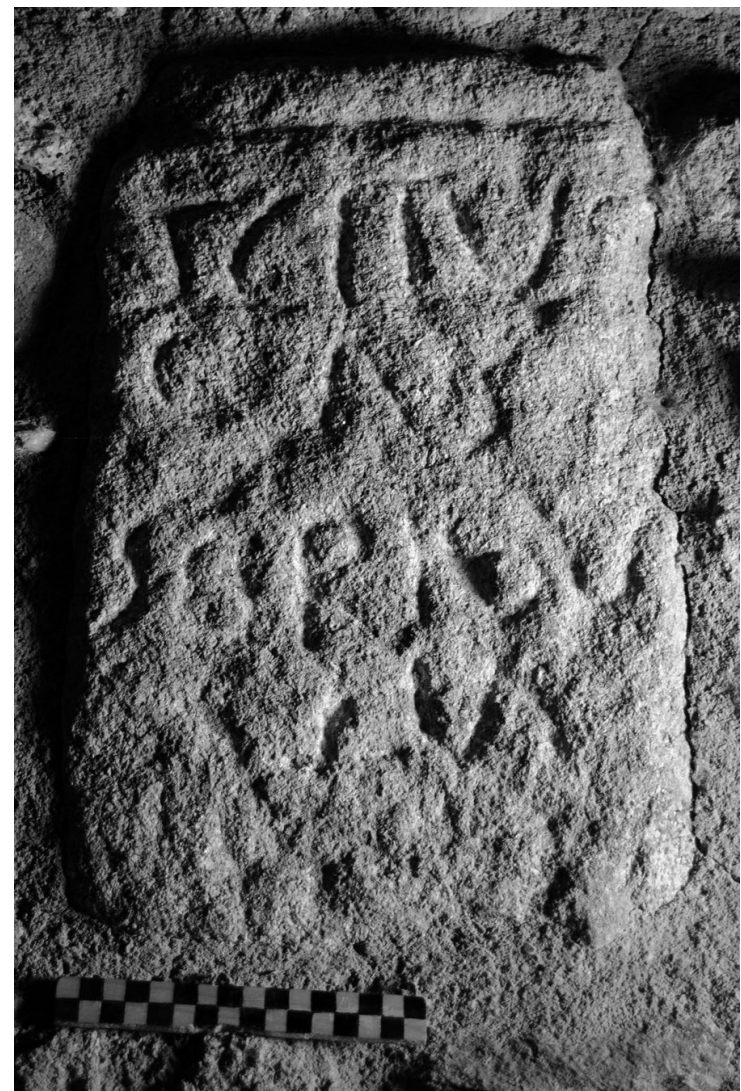

Figura 1. Foto: autor.

La lectura que proponemos es la siguiente:

$$
\text { Scitus / C+A+S(c. 1-2)/sḅrig̣o / ṿ(otum) s(olvit) I(ibens) a(nimo) }
$$

1. El presente trabajo se enmarca en el proyecto de investigación Sociedad romana y hábito epigráfico en la Hispania Citerior (HAR2015-65168-P), otorgado por el Ministerio de Economía y Competitividad de España. Por otra parte, queremos dar las gracias a Arcadio Marcos López y a Jesús Álvarez Sanchís, quienes nos dieron noticia de la existencia de la pieza. Asimismo, D. Arcadio Marcos nos explicó sobre el terreno las circunstancias del hallazgo de la misma. Igualmente, agradecemos a los evaluadores sus valiosas apreciaciones sobre el trabajo. 

Y NUEVA INTERPRETACIÓN DE TRES ARAS VOTIVAS EXTREMEÑAS

El nombre del dedicante es claro, Scitus, opción que goza de algunos paralelos seguros ${ }^{2}$ y que también podría estar testimoniado en un ara votiva hallada en Perales del Puerto ${ }^{3}$. En cualquier caso, el nombre personal Scitus es diáfano en la pieza.

No está tan claro el resto de la inscripción, a partir de 1. 2. Aquí hay desgastes importantes después de la primera letra y al final de la línea. Este renglón debía contener una posible filiación y, con más seguridad, el inicio del apelativo divino. No parece que se citara el teónimo además del apelativo, sino que la mención se reducía, probablemente, a este último. Esto viene dado por la probable terminación -brigo. No obstante, es posible que la línea 3 se pudiera leer serigo, lo que supondría también un apelativo divino, aunque no necesariamente vinculado con un topónimo. La fórmula votiva está algo desgastada, pero parece segura si exceptuamos el asta derecha de la V y está centrada, como es habitual en la epigrafía religiosa romana.

La pieza sería, por tanto, un altar dedicado a una divinidad autóctona, en una región donde no hay muchos monumentos de este tipo. En el entorno se conocen ofrendas votivas a las deidades vetonas Ilurbeda y $T o g a^{4}$, y a otras divinidades que solo conocemos por un testimonio. Con todo, sin duda el área vetona es una de las regiones donde el panteón religioso autóctono está, todavía, lejos de ser establecido con precisión.

\section{Altar depositado en el Museo Arqueológico Municipal de Badajoz}

El segundo monumento se trata de un altar de granito con zócalo y cabecera, trabajado a cuatro caras. La corona muestra pulvini laterales algo desgastados y fragmentados, y se une al fuste mediante dos toros que, en la actualidad, están también alterados. Del zócalo queda solo el rastro de la moldura. La cara superior no presenta focus. Las caras laterales muestran una banda vertical en relieve con una anchura de $3 \mathrm{~cm}$ que se extiende desde la cabecera hasta el zócalo. El campo epigráfico está dañado en su parte inferior izquierda, afectando al texto en 1. 5. Las dimensiones de la pieza son (69) x 36 × 33 cm y el campo epigráfico mide

2. $A E$ 2003, 709 (Montebello Vicentino, Vicenza); CIL V 8318 (Aquileia, Udine) y $A E$ 1984, 482 (Pousafoles do Bispo, Sabugal, Guarda); $A E$ 2010, 699 (Adeganha, Moncorvo, Bragança); $A E$ 1996, 856 (Idanha-a-Velha, Idanha-a-Nova, Castelo Branco); CIL II 1718 (Martos, Jaén); CIL II7 106 (Porcuna, Jaén); AE 2012, 20n (Rennes); CIL VI 3561 y 26011 (Roma); CIL X 4312 (Santa Maria Capua Vetere, Caserta); Kajanto 1965, 250.

3. Olivares Pedreño 2017, 174-175, n. ${ }^{\circ}$ 2, foto, fig. 3.

4. Id. 2001, 62-67; id. 2002, 50-51. 
Y NUEVA INTERPRETACIÓN DE TRES ARAS VOTIVAS EXTREMEÑAS

$42 \times 34 \mathrm{~cm}$. Las letras son rústicas, muy irregulares en cuanto a su forma y miden entre 5 y $6 \mathrm{~cm}$.

Se desconoce el lugar exacto de su hallazgo y está conservado en un almacén dependiente del Museo Arqueológico Provincial de Badajoz, en la misma ciudad.

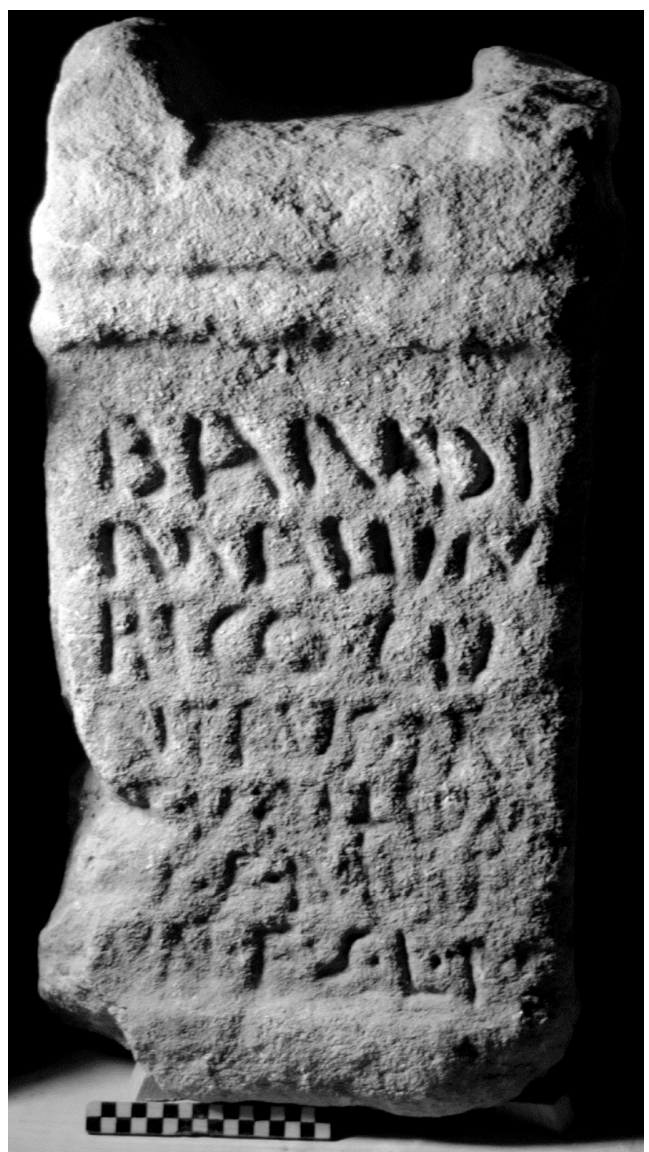

Figura 2. Museo de Badajoz (foto: autor).

Bandi / Malun/rico $\cdot \mathrm{Cu} / \mathrm{ntius} \cdot \mathrm{M}($ arci?) / [f(ilius)] $\cdot[S$ ? ]ại!c̣ọn/[u]s $f($ ecit) $\cdot$ Albịị/un̂us $\cdot f(i l i u s) \cdot s(o l v i t) \cdot \mid(i b e n s) \cdot m($ erito $)$

Ramírez Sádaba 1993, 436-437, n. ${ }^{\circ}$ 6, fig. 6, 1. 3: rico · Cu, 1. 5-7: $+u++i+s u+/ s f a+u /$ un $f$ s(olvit) l(ibens) m(erito) (AE 1994, 869; HEp 
5, 1995, 123); Prósper 1994, 194-195 (AE 1994, 819); Melena 1997, 377, 1. 4-5: ntius Max/sumi f(ilius), sin resolver el final de 1. 5; Salas, Esteban, Redondo y Sánchez Abal 1997, 77, n. ${ }^{\circ} 66$ (HEp 7, 1997, 176); Olivares Pedreño 2002, 35, lám. 4; Prósper 2002, 263.

El epíteto de la divinidad Bandus es Malunrico o Malunaico, contiene la raíz "mel- "salir, aparecer, mostrarse, ser elevado", presente en algunos topónimos de Hispania5. La propuesta de lectura de Prósper, Malunaico, es más probable fonéticamente, pero la $\mathrm{R}$ es más segura según la inscripción, a pesar de la irregularidad que presentan las letras. En este sentido, las A de 1.1 y 1.2 son diferentes a la posible A de 1.3.

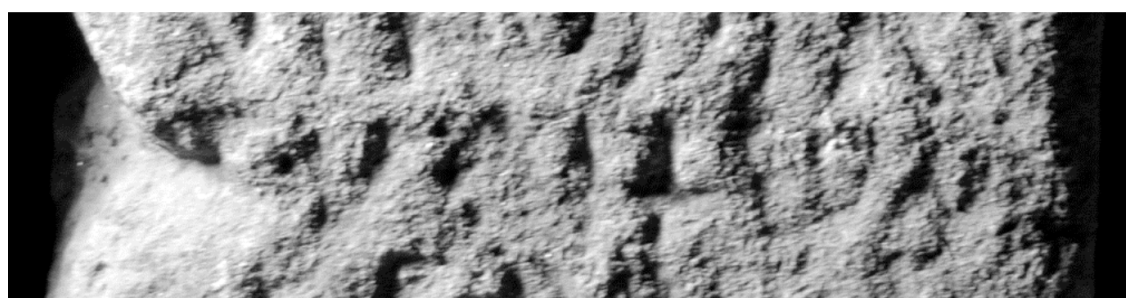

Figura 3. Lectura del cognomen [S]ailcon/[us].

Cuntius es conocido en Hispania por dos testimonios de Lusitania ${ }^{6}$. Las lecturas presentadas hasta el momento para las líneas 3-5 nos parecen revisables a tenor del texto visible. La lectura del cognomen, [S]ailconus o [SJailgonus, aunque no es segura, es bastante probable a partir de la observación de la pieza. Lo más probable es que el nombre sea Sailconus, ya que antes de la letra $a$ aparece una letra muy semejante a la $s$ de 1 . 4. Antropónimos relacionados con el que nos ocupa existen algunos en Lusitania $^{7}$, como Sailcius ${ }^{8}$, Saelgius ${ }^{9}$, Sailgius ${ }^{10}$ o Saulcius ${ }^{11}$. También conocemos el gentilicio Sailcieicon en Yecla de Yeltes (Salamanca) ${ }^{12}$, aunque es dudoso.

5. Pedrero 2001, 550-551.

6. Abascal 1994, 126; Vallejo 2005, 298-299.

7. Estos antropónimos están recogidos por VAllejo 2005, 391.

8. Encarnação 1975, 175-176; García 1991, 355, n. ${ }^{\circ}$ 201; Vallejo 2005, 391 (Freixo de Numão, Guarda).

9. Hernández Guerra 2001, 24-25, n. ${ }^{\circ} 13$ (Martiago, Salamanca)

10. Esteban 2012, 36-36, n. 428 (Abertura, Cáceres); id. 2016, 26-28, n. 1162 (Casas de Millán, Cáceres) y CIL II 427 (Prados, Guarda).

11. VAllejo 2005, 391.

12. CIL II 5314; Hernández Guerra 2001, 150, n. ${ }^{\circ} 173$. 

Y NUEVA INTERPRETACIÓN DE TRES ARAS VOTIVAS EXTREMEÑAS

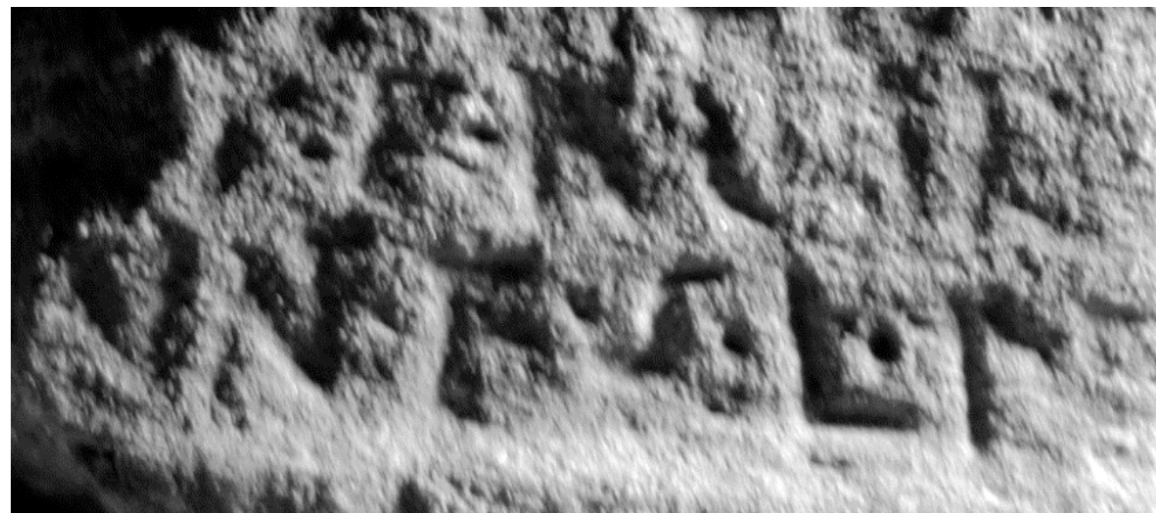

Figura 4. Lectura del nombre Albir/unus.

También es dudoso el nombre del individuo que llevó a cabo la erección del monumento, Albirunus o Albilunus, aunque uno de estos dos también es bastante probable. Este nombre, a pesar de tener paralelos cercanos en la onomástica latina, también podría ser una forma autóctona ${ }^{13}$. Con todo, los dos nombres son desconocidos hasta el momento en la epigrafía del Imperio romano. Esta inscripción se añade a otras muchas aras que han generado cierta confusión interpretativa por la aparición del nombre del individuo que hizo el voto junto al del que lo lleva a cabo ${ }^{14}$. En estos casos, suele aparecer el verbo fecit y la referencia al parentesco entre ambos, pero el texto adquiere una estructura inhabitual que, en casos de desgaste de los monumentos, suele plantear serios obstáculos interpretativos.

\section{Ara procedente de Salvatierra de los Barros (Badajoz)}

El ara es de granito de grano grueso, con zócalo y cabecera, y está trabajada a cuatro caras. La cara superior es lisa. La parte derecha del zócalo está fracturada y también fue rebajado, en parte, todo el lado derecho del fuste y el capitel. Sus medidas máximas son 85 x (41) x 27,5 y las letras son capitales, con medidas de $4,5 \mathrm{~cm}$. Las interpunciones son circulares.

Fue hallada en 1994 entre los escombros de la ermita de Santa Lucía, de probable origen visigodo, situada junto a la carretera que se dirige a

13. Vid. Vallejo 2005, 123.

14. Olivares 2019, 152 y ss. 
Burguillos. Este contexto tiene interesantes semejanzas con el de los altares de Ataecina hallados en la ermita visigoda de Santa Lucía del Trampal, en Alcuéscar (Cáceres). La pieza se halla depositada en el Museo municipal de cerámica de Salvatierra de los Barros, donde la pudimos estudiar y fotografiar el 3 de mayo de 2019.

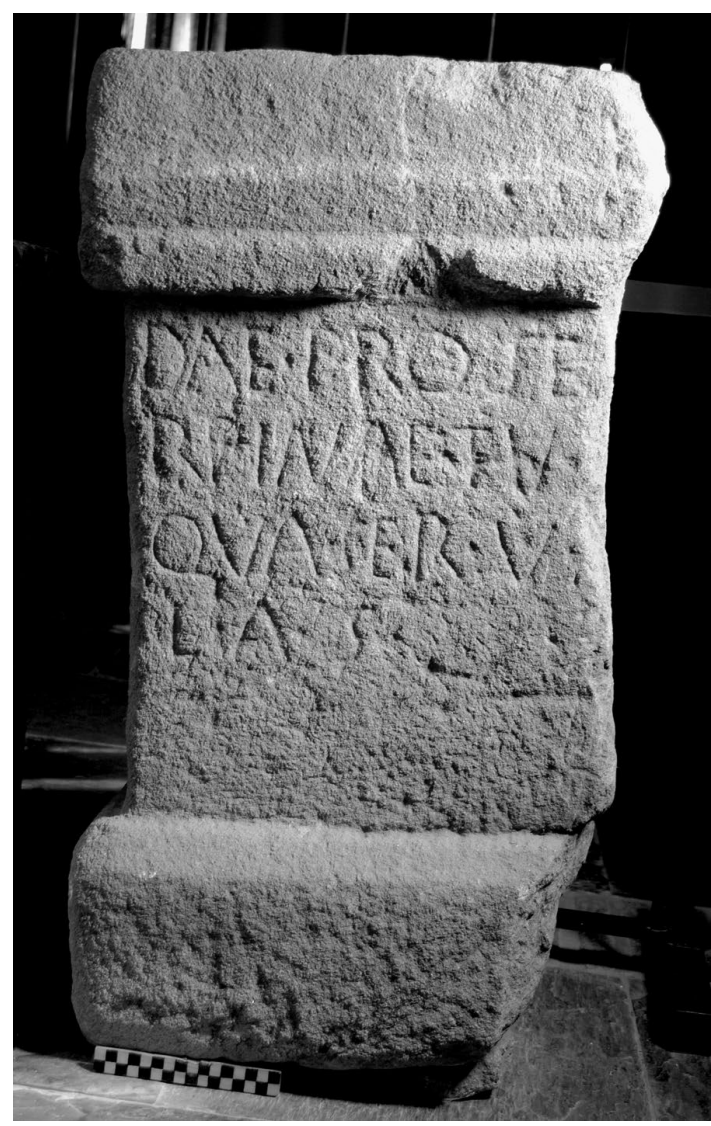

Figura 5. Museo de la alfarería de Salvatierra de los Barros (foto: autor). s(olvit) .

D(e)ae $\cdot$ Prose/rpinae $\cdot$ Tu/qua $\cdot \operatorname{ser}(v a) \cdot v($ otum $) /$ (ibens) $\cdot$ a(nimo) $\cdot$

Abascal 1995, 89, n. ${ }^{\circ}$, nota 302 (HEp 6, 1996, 142); Canto 1997, 96, n. ${ }^{\circ}$ 89, foto 56, 1. 1-3: D(eae) - At(aecinae) Prose/rpinae - Tur(obrigensi) . / Qua(---) · ser(vus vel -va) · v(otum) · (AE 1997, 815); Prósper 2002, 290. 
Y NUEVA INTERPRETACIÓN DE TRES ARAS VOTIVAS EXTREMEÑAS

Incluimos esta pieza porque fue interpretada por Canto como una ofrenda a Ataecina. Ello viene dado por un error de lectura en 1. 1, puesto que la lectura $D(e) a e$ es fiable y no es procedente la hipótesis $D(e a e)$ At(aecinae) puesto que no existe la $\mathrm{T}$, sino una $\mathrm{E}$ muy clara. Al final de 1. 2 tampoco se lee Tur, como afirmaba Canto, sino Tu, debiendo considerarse como el inicio del nombre de la esclava: Tuqua. Al final de 1. 2 se afirmaba la existencia de una interpunción, pero es, probablemente, un pequeño desconche como los existentes a la derecha de la $\mathrm{T}$ o en medio de la V de la misma línea. Esta hipótesis responde a la lectura y es totalmente coherente teniendo en cuenta la estructura habitual de las ofrendas votivas. La opción de Canto era, en verdad, muy extraña, puesto que requería que el nombre de Ataecina estuviera abreviado, mientras el de Proserpina se exponía en su totalidad. Además, suponía admitir el apelativo Turobrigensis abreviado y situado después del teónimo romano, lo cual también era difícil de aceptar, por no existir paralelos. En definitiva, creemos que el presente monumento debe ser eliminado de los catálogos relativos a la diosa lusitana Ataecina.

La dedicante sería, por tanto, una esclava, Tuqua, aunque no tenemos este nombre testimoniado hasta la fecha.

\section{Ara hallada en Santa Ana (Cáceres) dedicada a Adegina}

Se trata de un ara en granito rosado de la que se ha perdido el zócalo y parte del fuste. También ha perdido los elementos superiores de su cabecera, aunque conserva líneas incisas paralelas que parecen delimitar el espacio respecto al fuste. Las medidas totales de la pieza son (43) x 28 $\mathrm{cm}$. Las letras son rústicas y no están debidamente alineadas.

Se desconoce su procedencia. Actualmente está empotrada a 3 metros de altura en la pared de la casa . $^{\circ} 15$ de la carretera de Robledillo de Trujillo, a la izquierda de la puerta principal, dentro del núcleo urbano de Santa Ana, donde la pudimos estudiar y fotografiar el 4 de mayo de 2019 . 


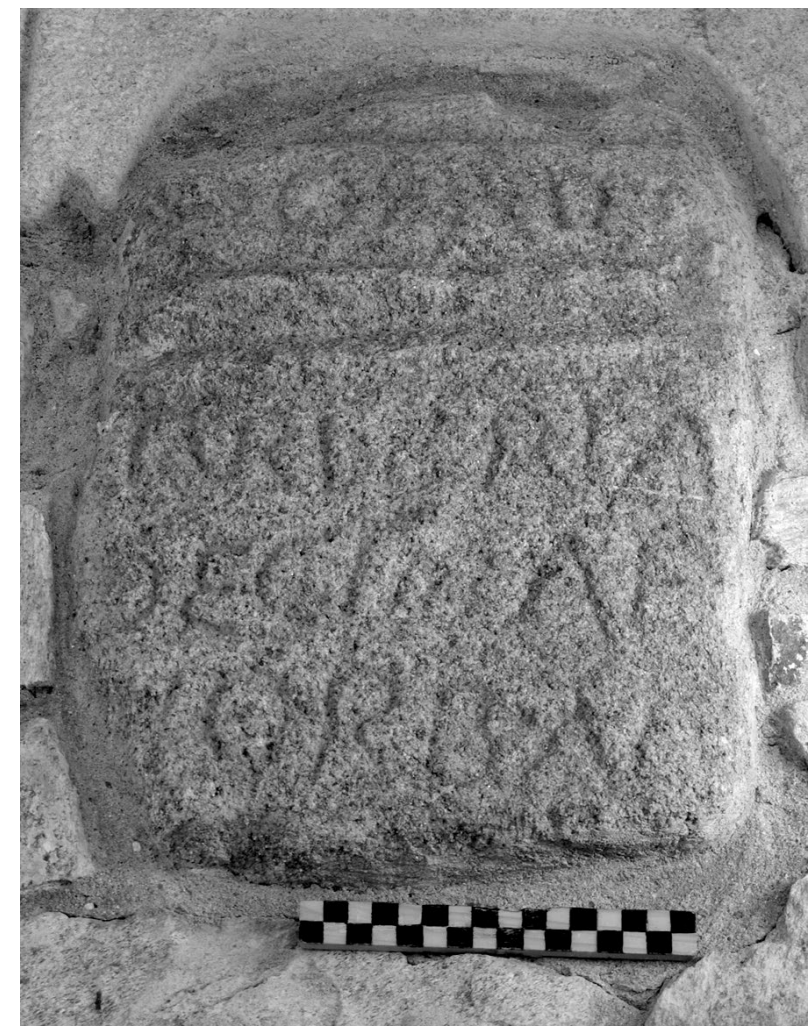

Figura 6. Foto: autor.

\section{Domin(ae) / Turibri(gensi) A/deginae / Ṇorbâṇ/}

Esteban 2007, 224, n. 321 , foto y p. 415, lám. 321, 1. 1-3: Dominae / Turibrig(ensi) A/deginae (HEp 16, 2007, 130).

Las variantes que proponemos para esta pieza son más de matiz, pero son patentes en la pieza y no parece que sean producto de un desgaste desde el estudio realizado anteriormente. Se trata de la categoría religiosa Domin(ae), que no aparece completa, como había establecido Esteban. Tampoco el apelativo de la diosa se cita como estableció el citado autor, puesto que la $G$ no está presente en la pieza, apareciendo como Turibri(gensi). Finalmente, el teónimo no lleva un nexo NA, ya que las dos letras se ven separadas con claridad. En la última línea, seguimos la lectura de Esteban. 
5. Bibliografía

Abascal, J. M. (1994): Los nombres personales en las inscripciones latinas de Hispania, Madrid-Murcia.

Abascal, J. M. (1995): "Las inscripciones latinas de Santa Lucía del Trampal (Alcuéscar, Cáceres) y el culto de Ataecina en Hispania", AEspA 68, pp. 31-105.

Canto, A. (1997): Epigrafía Romana de la Beturia Céltica, Madrid.

EnCARnAÇÃo, J. (1975): Divindades indigenas sob o dominio romano em Portugal, Lisboa.

Esteban, J. (2007): Corpus de inscripciones latinas de Cáceres I. Norba, Cáceres.

Esteban, J. (2012): Corpus de inscripciones latinas de Cáceres II. Turgalium, Cáceres.

Esteban, J. (2016): Corpus de inscripciones latinas de Cáceres IV. Caurium, Cáceres.

García, J. M. (1991): Religiões antigas de Portugal. Aditamentos e observações as "Religiões da Lusitania" de J. Leite de Vasconcelos, Lisboa.

Hernández Guerra, L. (2001): Epigrafía de época romana de la provincial de Salamanca, Valladolid.

Kajanto, I. (1982): The Latin Cognomina, Roma (1. ${ }^{\text {a }}$ ed., Helsinki, 1965).

Melena, J. L. (1997): "Notas de epigrafía romana de Extremadura: III. Sobre un nuevo nexo de letras», Veleia 14, pp. 375-378.

Olivares Pedreño, J. C. (2001): «Teónimos y pueblos indígenas hispanos: los vettones», Iberia 4, pp. 57-69.

Olivares Pedreño, J. C. (2002): Los dioses de la Hispania Céltica, Madrid.

Olivares Pedreño, J. C. (2017): «Correcciones y nuevas interpretaciones sobre siete inscripciones votivas de Lusitania", Habis 48, pp. 171-185.

Olivares pedreño, J. C. (2019): "Revisión de seis inscripciones en altares votivos dedicados a deidades lusitanas y vetonas", Veleia 36, pp. 149162.

Pedrero, R. (2001): "Los epítetos del teónimo occidental Bandue/i», en F. Villar y M. P. Fernández Álvarez (eds.): Religión, lengua y cultura prerromanas de Hispania, Salamanca, pp. 541-560.

Prósper, B. (1994): "El teónimo paleohispano Trebarune», Veleia 11, pp. 187-196.

PRÓsper, B. (2002): Lenguas y religiones prerromanas del occidente de la Península ibérica, Salamanca.

RAMírez SÁDABA, J. L. (1993): "Teónimo y Antropónimos nuevos en la provincia de Lusitania y zonas próximas", en Lengua y cultura en la Hispania prerromana. Actas del $V$ coloquio sobre lenguas y culturas prerroma- 
nas de la Península ibérica (Colonia, 25-28 de noviembre de 1989), Salamanca, pp. 425-444.

Salas, J.; Esteban, J.; Redondo, J. A. y Sánchez Abal, J. L. (1997): Inscripciones romanas y cristianas del Museo Arqueológico Provincial de Badajoz, Badajoz.

VAllejo, J. M. (2005): Antroponimia indígena de la Lusitania romana, Vitoria. 